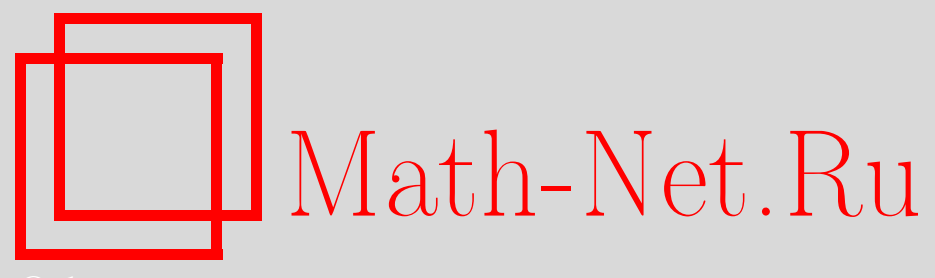

С. С. Марченков, Клоны, определяемые знакопеременными моноидами, Дискрет. матем., 2002, том 14, выпуск 2, 3-8

DOI: https://doi.org/10.4213/dm236

Использование Общероссийского математического портала Math-Net.Ru подразумевает, что вы прочитали и согласны с пользовательским соглашением http: //www.mathnet.ru/rus/agreement

Параметры загрузки:

IP: 18.209 .158 .208

26 апреля 2023 г., 14:44:01

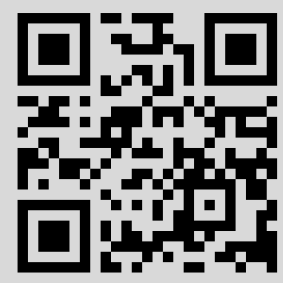




\title{
Клоны, определяемые знакопеременными моноидами
}

\author{
() 2002 г. С. С. Марченков
}

\begin{abstract}
Для любого $k, k \geqslant 3$, в симметрической полугруппе $\mathscr{T}_{k}$ всех подстановок на множестве $E_{k}$ рассматриваются знакопеременные моноиды - моноиды, которые содержат все четные перестановки на $E_{k}$. Для каждого моноида $T$ из $\mathcal{T}_{k}$ определяется множество всех функций из $P_{k}$, которые сохраняют графики всех подстановок из $T$ (клон, определяемый моноидом $T$ ). Описываются все клоны, определяемые знакопеременными моноидами.

Работа выполнена при поддержке Российского фонда фундаментальных исследований, проект 00-01-00351.
\end{abstract}

Известно [1], что при $k \geqslant 3$ классификация множества $P_{k}$ функций $k$-значной логики, основанная на операции суперпозиции, континуальна. В связи с этим появились различные подходы к построению эффективных классификаций множества $P_{k}$, использующие алгебраические, логические и программистские идеи. Одна из таких идей состоит в том, чтобы замкнутые классы определять с использованием групп или полугрупп симметрий (групп автоморфизмов, полугрупп эндоморфизмов).

Для функции $f$ из $P_{k}$ группа симметрий - это группа автоморфизмов алгебры $\left\langle E_{k}, f\right\rangle$, полугруппа симметрий - полугруппа эндоморфизмов алгебры $\left\langle E_{k}, f\right\rangle$. Возникает вопрос: если задана группа (полугруппа) симметрий, то как выглядит множество всех функций, имеющих данную группу (полугруппу) симметрий?

Удовлетворительный ответ на этот вопрос получен лишь в немногих случаях. Так, довольно хорошо изучен класс однородных функций [2], отвечающий полной симметрической группе перестановок $\mathscr{S}_{k}$, а также класс четных функций $[3,4]$, который соответствует знакопеременной подгруппе $\mathscr{A}_{k}$ группы $\mathscr{Y}_{k}$.

В [5] сформулирована задача об описании клонов (замкнутых классов, содержащих селекторные функщии), которые определяются моноидами (полугруппами с единицей), содержащими группу $\mathscr{S}_{k}$, и сделаны первые шаги в направлении ее решения. Отметим, что практически все полученные в [5] результаты являются следствиями результатов из работ [6-10]. Оказалось, что этот вопрос тесно связан с классификацией однородных функций, которая построена в [6-8]. В настоящей заметке мы рассматриваем более общую задачу, когда вместо группы $\mathscr{S}_{k}$ берется группа $\mathscr{A}_{k}$. На основе классификации четных функщий, полученной в $[3,4,11-13]$, мы даем полное решение этой задачи.

Пусть $k$ - натуральное число, $k \geqslant 3, E_{k}=\{0,1, \ldots, k-1\}, P_{k}^{(n)}-$ множество всех $n$-местных функций, отображающих $E_{k}^{n}$ в $E_{k}, P_{k}=\bigcup_{n=1}^{\infty} P_{k}^{(n)}$. Через $O_{k}$ обозначим 
множество всех селекторных функщий (проекщий) из $P_{k}$, то есть функщий $e_{i}^{n}$, удовлетворяющих тождествам

$$
e_{i}^{n}\left(x_{1}, \ldots, x_{i}, \ldots, x_{n}\right)=x_{i}, \quad 1 \leqslant i \leqslant n, \quad n=1,2 \ldots
$$

На множестве $P_{k}$ считаем заданной операцию суперпозиции (см. [14]). Подмножества множества $P_{k}$, замкнутые относительно операции суперпозиции, называем замкнутыми классами. Замкнутые классы, содержащие все селекторные функции, носят название клонов.

Через $\mathscr{T}_{k}$ обозначим симметрическую полугруппу всех отображений из $E_{k}$ в $E_{k}$ с операцией композиции (суперпозиции) отображений, через $\mathscr{S}_{k}-$ симметрическую групту всех перестановок на множестве $E_{k}$, а через $\mathscr{A}_{k}$ - знакопеременную подгруппу группы $\mathscr{S}_{k}$.

Под $m$-местным отношением на множестве $E_{k}$ понимаем произвольное подмножество множества $E_{k}^{m}$. Если $f-$ функция из $P_{k}^{(1)}$, то графиком функции $f$ называем бинарное отношение $\left\{(x, f(x)): x \in E_{k}\right\}$. График функции $f$ обозначаем через $f^{\square}$.

Пусть $f \in P_{k}^{(n)}, \rho-m$-местное отношение на $E_{k}$. Говорят, что функция $f$ сохраняет отношение $\rho$, если для любых $n$ наборов $\left(a_{11}, \ldots, a_{m 1}\right), \ldots,\left(a_{1 n}, \ldots, a_{m n}\right)$, принадлежащих отношению $\rho$, набор $\left(f\left(a_{11}, \ldots, a_{1 n}\right), \ldots, f\left(a_{m 1}, \ldots, a_{m n}\right)\right)$ также принадлежит отношению $\rho$. Множество всех функций, сохраняющих отношение $\rho$, обозначим через $\operatorname{Pol}(\rho)$. Хорошо известно (см., например, [15]), что для любого отношения $\rho$ множество $\operatorname{Pol}(\rho)$ является клоном.

Если $f \in P_{k}^{(n)}, s \in \mathscr{T}_{k}$ и выполняется тождество

$$
f\left(s\left(x_{1}\right), \ldots, s\left(x_{n}\right)\right)=s\left(f\left(x_{1}, \ldots, x_{n}\right)\right),
$$

то говорят, что $s$ есть эндоморфизм алгебры $\left\langle E_{k}, f\right\rangle$. Условие (1) эквивалентно также тому, что функция $f$ сохраняет отношение $s$. Множество $\operatorname{End}\left\langle E_{k}, f\right\rangle$ всех эндоморфизмов алгебры $\left\langle E_{k}, f\right\rangle$ образует моноид - полугруппу с единицей (тождественным отображением). Аналогичным образом, если $s \in \mathscr{S}_{k}$ и выполняется тождество (1), то $s$ называют автоморфизмом алгебры $\left\langle E_{k}, f\right\rangle$. Множество Aut $\left\langle E_{k}, f\right\rangle$ всех автоморфизмов алгебры $\left\langle E_{k}, f\right\rangle$ образует подгруппу симметрической группы $\mathscr{S}_{k}$.

Если $s \in \mathscr{T}_{k}$, то для всякой функщии $f$ из $\operatorname{Pol}\left(s^{\square}\right)$ моноид $\operatorname{End}\left\langle E_{k}, f\right\rangle$ содержит, очевидно, элемент $s$. Для любого моноида $T, T \subseteq \mathscr{T}_{k}$, положим

$$
\varphi(T)=\bigcap_{s \in T} \operatorname{Pol}\left(s^{\square}\right) .
$$

Из определения следует, что для любой функции $f$ из $\varphi(T)$ выполняется соотношение $T \subseteq \operatorname{End}\left\langle E_{k}, f\right\rangle$. Таким образом, $\varphi(T)$ есть множество всех функций из $P_{k}$, у которых полугруппа эндоморфизмов $\operatorname{End}\left\langle E_{k}, f\right\rangle$ содержит моноид $T$.

В [5] сформулирована задача об описании клонов $\varphi(T)$ для случая, когда $\mathscr{S}_{k} \subseteq T$. Ниже мы полностью решаем более общую задачу, когда моноид $T$ содержит знакопеременную группу $\mathscr{A}_{k}$. Такие моноиды $T$ мы называем знакопеременными.

Следуя [2], назовем функцию $f$ из $P_{k}$ однородной, если $\operatorname{Aut}\left\langle E_{k}, f\right\rangle=\mathscr{S}_{k}$. Множество всех однородных функций из $P_{k}$ обозначим через $S_{k}$. В $[3,4]$ функция $f$ из $P_{k}$ названа четной, если $\mathscr{A}_{k} \subseteq \mathrm{Aut}\left\langle E_{k}, f\right\rangle$. Множество всех четных функций из $P_{k}$ обозначим через $A_{k}$. В [6-8] для любого $k, k \geqslant 3$, описаны все клоны однородных функций из $P_{k}$, а в $[3,4]-$ все клоны четных функций из $P_{k}$ при $k \geqslant 4$ (в случае $k=3$ число клонов четных функщий континуально [16]). Кроме того, в [11-13] описан счетный фрагмент решетки клонов четных функций из $P_{3}$. 
Напомним определение некоторых однородных и четных функций. При любом $k$, $k \geqslant 3$, следующие функции $d(x, y, z)$ и $l_{k}\left(x_{1}, \ldots, x_{k}\right)$ из $P_{k}$ однородны:

$$
\begin{aligned}
d(x, y, z) & = \begin{cases}x, & \text { если } x=y, \\
z, & \text { если } x \neq y,\end{cases} \\
l_{k}\left(x_{1}, \ldots, x_{k}\right) & = \begin{cases}x_{1}, & \text { если значения } x_{1}, \ldots, x_{k} \text { попарно различны, } \\
x_{k} & \text { в противном случае. }\end{cases}
\end{aligned}
$$

В классе $P_{3}$ однородной является функщия $2 x+2 y(\bmod 3)$, а в классе $P_{4}-$ функция $x+y+z$, где сложения (из поля Галуа $G F(4))$ определяется таблицей

\begin{tabular}{|c|cccc|}
\hline+ & 0 & 1 & 2 & 3 \\
\hline 0 & 0 & 1 & 2 & 3 \\
1 & 1 & 0 & 3 & 2 \\
2 & 2 & 3 & 0 & 1 \\
3 & 3 & 2 & 1 & 0 \\
\hline
\end{tabular}

В классе $P_{3}$ четными являются функции $x+1(\bmod 3), \alpha(x, y)$ и $\beta(x, y)$, где функции $\alpha$ и $\beta$ определяются таблицами

\begin{tabular}{|c|ccc|}
\hline$\alpha$ & 0 & 1 & 2 \\
\hline 0 & 0 & 1 & 0 \\
1 & 1 & 1 & 2 \\
2 & 0 & 2 & 2 \\
\hline
\end{tabular}

\begin{tabular}{|l|lll|}
\hline$\beta$ & 0 & 1 & 2 \\
\hline 0 & 0 & 0 & 2 \\
1 & 0 & 1 & 1 \\
2 & 2 & 1 & 2 \\
\hline
\end{tabular}

Так же, как в $[7,8]$, через $S_{4}^{4} L_{4}$ обозначим клон всех однородных функций из $P_{4}$, линейных над полем $G F(4)$. Через $A_{3}^{0}$ обозначим клон всех четных функщий из $P_{3}$, сохраняющих константу 0 (в [11-13] клон $A_{3}^{0}$ имеет обозначение $S_{1}$ ).

Для любого $k, k \geqslant 3$, обозначим через $T_{k}^{a}$ моноид, состоящий из всех перестановок группы $\mathscr{A}_{k}$ и всех констант, а через $T_{k}^{s}$ - моноид, который состоит из всех перестановок группы $\mathscr{S}_{k}$ и всех констант. Пусть $T_{4}^{l}$ обозначает моноид, который состоит из всех перестановок группы $\mathscr{A}_{4}$, всех констант, а также всех функций $f(x)$, которые удовлетворяют следующему условию: область значений функции $f$ состоит из двух элементов $b_{1}, b_{2}$, причем

$$
\left|f^{-1}\left(b_{1}\right)\right|=\left|f^{-1}\left(b_{2}\right)\right|=2,
$$

где через $\left|f^{-1}\left(b_{i}\right)\right|$ обозначено число элементов множества $f^{-1}\left(b_{i}\right)$.

Теорема 1. Имеют место равенства

$$
\begin{array}{llc}
\varphi\left(\mathscr{A}_{k}\right)=A_{k}, & \varphi\left(T_{k}^{a}\right)=A_{k}, & k \geqslant 4, \\
\varphi\left(T_{3}^{a}\right)=A_{3}^{0}, & \varphi\left(\mathscr{S}_{k}\right)=\varphi\left(T_{k}^{s}\right)=S_{k}, & \varphi\left(T_{4}^{l}\right)=S_{4}^{4} L_{4} .
\end{array}
$$

Для всех остальных знакопеременных моноидов $T, T \subseteq \mathscr{T}_{k}$, выполняется равенство $\varphi(T)=O_{k}$.

Доказательство. Равенства

$$
\varphi\left(\mathscr{A}_{k}\right)=A_{k}, \quad \varphi\left(T_{3}^{a}\right)=A_{3}^{0}, \quad \varphi\left(\mathscr{S}_{k}\right)=S_{k}
$$


следуют из определения клонов $A_{k}, A_{3}^{0}$ и $S_{k}$. При $k \geqslant 4$ равенство $\varphi\left(T_{k}^{a}\right)=A_{k}$ следует из того, что при $k \geqslant 4$ любая четная функщия из $P_{k}$ сохраняет любую константу (см. $[3,4])$, а равенство $\varphi\left(T_{k}^{s}\right)=S_{k}-$ из того, что при $k \geqslant 3$ любая однородная функция из $P_{k}$ также сохраняет любую константу (см. [2]). Поскольку каждый знакопеременный моноид, содержащий константу, очевидным образом содержит все константы, мы тем самым рассмотрели все знакопеременные моноиды, которые состоят из перестановок и еще, быть может, констант. Поэтому далее будем рассматривать случаи, когда знакопеременный моноид $T, T \subseteq \mathscr{T}_{k}$, содержит функщию $f(x)$, отличную от перестановки и константы. В этом случае в множестве $E_{k}$ найдутся такие попарно различные элементы $a_{1}, a_{2}, a_{3}$ и неравные элементы $b_{1}, b_{2}$, что

$$
f\left(a_{1}\right)=f\left(a_{2}\right)=b_{1}, \quad f\left(a_{3}\right)=b_{2} .
$$

Основная часть оставшегося доказательства опирается на следующий факт, который вытекает из результатов работ $[3,4,6-8,11-13]$.

Любой клон четных функций из $P_{k}$, отличный от клона $O_{k}$, содержит хотя бы одну из следующий функщий: $d(x, y, z), l_{k}\left(x_{1}, \ldots, x_{k}\right), x+y+z(k=4$, сложение из поля $G F(4))$, $x+1,2 x+2 y, \alpha(x, y), \beta(x, y)$ (все четыре функции из $P_{3}$, сложение по модулю 3 ).

Покажем, что ни одна из функций $d, l_{k}, x+1,2 x+2 y, \alpha, \beta$ не сохраняет отношение $f^{\square}$ для функщии $f$, удовлетворяющей условиям (2). Начнем с функщии $d$. Очевидно, что пары $\left(a_{1}, b_{1}\right),\left(a_{2}, b_{1}\right),\left(a_{3}, b_{2}\right)$ принадлежат отношению $f^{\square}$, пара $\left(a_{3}, b_{1}\right)$ не принадлежит отношению $f^{\square}$ и

$$
d\left(a_{1}, a_{2}, a_{3}\right)=a_{3}, \quad d\left(b_{1}, b_{1}, b_{2}\right)=b_{1} .
$$

Отсюда вытекает, что функция $d$ не сохраняет отношение $f^{\square}$.

Обратимся к функщии $l_{k}$. Пусть

$$
\left\{a_{4}, \ldots, a_{k}\right\}=E_{k} \backslash\left\{a_{1}, a_{2}, a_{3}\right\}, \quad b_{4}=f\left(a_{4}\right), \ldots, b_{k}=f\left(a_{k}\right) .
$$

Тогда наборы

$$
\left(a_{1}, b_{1}\right),\left(a_{2}, b_{1}\right),\left(a_{4}, b_{4}\right), \ldots,\left(a_{k}, b_{k}\right),\left(a_{3}, b_{2}\right)
$$

принадлежат отношению $f^{\square}$, набор $\left(a_{1}, b_{2}\right)$ не принадлежит отношению $f^{\square}$ и

$$
l_{k}\left(a_{1}, a_{2}, a_{4}, \ldots, a_{k}, a_{3}\right)=a_{1}, \quad l_{k}\left(b_{1}, b_{1}, b_{4}, \ldots, b_{k}, b_{2}\right)=b_{2} \text {. }
$$

Это означает, что функция $l_{k}$ не сохраняет отношение $f^{\square}$.

Пусть $k=3$. Очевидно, что для элементов $a_{1}, a_{2}$ по модулю 3 выполняется одно из соотношений $a_{2}=a_{1}+1$ или $a_{1}=a_{2}+1$. Так как $f\left(a_{1}\right)=f\left(a_{2}\right)$, отсюда сразу вытекает, что функция $x+1$ не сохраняет отношение $f^{\square}$.

Далее, поскольку по модулю 3 справедливы равенства

$$
2 a_{1}+2 a_{2}=a_{3}, \quad 2 b_{1}+2 b_{1}=b_{1},
$$

а набор $\left(a_{3}, b_{1}\right)$ не принадлежит отношению $f^{\square}$, получаем, что функция $2 x+2 y$ не сохраняет отношение $f^{\square}$.

Перейдем к рассмотрению функций $\alpha(x, y)$ и $\beta(x, y)$. Можно предполагать, что

$$
\left\{a_{1}, a_{2}\right\}=\left\{b_{1}, b_{2}\right\}=\{0,1\}, \quad a_{3}=2 .
$$


В самом деле, если, например, $\left\{a_{1}, a_{2}\right\} \neq\{0,1\}$, то выберем в группе $\mathscr{A}_{3}$ такую перестановку $f_{1}$, которая отображает множество $\{0,1\}$ на множество $\left\{a_{1}, a_{2}\right\}$. Тогда для функции $f^{\prime}(x)=f\left(f_{1}(x)\right)$, принадлежащей моноиду $T$, получим, что

$$
f^{\prime}(0)=f^{\prime}(1)=b_{1}, \quad f^{\prime}(2)=b_{2} .
$$

Аналогичным образом поступаем в случае, когда $\left\{b_{1}, b_{2}\right\} \neq\{0,1\}$.

Итак, пусть справедливы соотношения (3). Если $b_{1}=0$, то отношению $f^{\square}$ принадлежат наборы $(0,0),(2,1)$ и не принадлежит набор $(0,1)$. Поскольку $\alpha(0,2)=0$ и $\alpha(0,1)=1$, получаем, что функция $\alpha$ в данном случае не сохраняет отношение $f^{\square}$. При условии $b_{1}=1$ следует выбрать наборы $(1,1),(2,0)$, принадлежащие отношению $f^{\square}$, и набор $(2,1)$, который не принадлежит отношению $f^{\square}$.

При рассмотрении функции $\beta$ можно, например, воспользоваться тем, что $\beta$ двойственна функции $\alpha$ относительно перестановки $s(x)=2 x+1(\bmod 3)$, то есть

$$
\beta(x, y)=s^{-1}(\alpha(s(x), s(y))),
$$

а при переходе к двойственным функциям необходимые нам свойства функции $f$ сохраняются, поскольку перестановка $2 x+1$ отображает множество $\{0,1\}$ на себя.

Итак, если в моноид $T$ входит функция $f$, которая удовлетворяет условиям (2) для множеств $\left\{a_{1}, a_{2}, a_{3}\right\},\left\{b_{1}, b_{2}\right\}$ с попарно различными элементами, то клон $\varphi(T)$ не может содержать ни одну из функций $d, l_{k}, x+1,2 x+2 y, \alpha, \beta$. Это означает, что в данном случае при $k \neq 4$ справедливо равенство $\varphi(T)=O_{k}$.

Остается исследовать возможность сохранения отношения $f^{\square}$ функщией $x+y+z$ (сложение из поля $G F(4)$ ).

Пусть сначала для элемента $a_{4}$, где $\left\{a_{4}\right\}=E_{4} \backslash\left\{a_{1}, a_{2}, a_{3}\right\}$, выполняется неравенство $f\left(a_{4}\right) \neq b_{2}$. Тогда наборы $\left(a_{1}, b_{1}\right),\left(a_{2}, b_{1}\right),\left(a_{3}, b_{2}\right)$ принадлежат отношению $f^{\square}$, набор $\left(a_{4}, b_{2}\right)$ не принадлежит отношению $f^{\square}$ и

$$
a_{1}+a_{2}+a_{3}=a_{4}, \quad b_{1}+b_{1}+b_{2}=b_{2} .
$$

Следовательно, функция $x+y+z$ в этом случае не сохраняет отношение $f^{\square}$.

Пусть теперь для указанного элемента $a_{4}$ справедливо равенство $f\left(a_{4}\right)=b_{2}$. Тогда соотношения (4) и аналогичные им соотношения

$$
a_{1}+a_{2}+a_{4}=a_{3}, \quad a_{1}+a_{3}+a_{4}=a_{2}, \quad a_{2}+a_{3}+a_{4}=a_{1}, \quad b_{1}+b_{2}+b_{2}=b_{1}
$$

показывают, что функция $x+y+z$ сохраняет отношение $f^{\square}$. Значит, в этом случае клон $\varphi(T)$ содержит функцию $x+y+z$. Однако функция $x+y+z$ порождает (в смысле операции суперпозиции) клон $S_{4}^{4} L_{4}$ (см. [7,8]). Следовательно, $S_{4}^{4} L_{4} \subseteq \varphi(T)$. Если моноид $T \subseteq \sigma_{4}$ содержит некоторую функцию $f$ такую, что $\left|f^{-1}\left(b_{1}\right)\right|=\left|f^{-1}\left(b_{2}\right)\right|=2$, то, как показано в $[17,18]$, он содержит также все функции этого типа. Кроме того, моноиду $T$ в этом случае принадлежат все константы. Если никакие другие функции (помимо перестановок из $\mathscr{A}_{4}$ ) в моноид $T$ не входят, то, очевидно, $\varphi(T)=S_{4}^{4} L_{4}$. В противном случае, как установлено выше, клону $\varphi(T)$ не принадлежит функция $x+y+z$ и, значит, он совпадает с клоном $\mathrm{O}_{4}$. Теорема доказана. 


\section{Список литературы}

1. Янов Ю. И., Мучник А. А., О существовании $k$-значных замкнутых классов, не имеющих конечного базиса. Докл. АН СССР (1959) 127, №1, 44-46.

2. Marczewski E., Homogeneous algebras and homogeneous operations. Fund. Math. (1964) 56, 81103.

3. Марченков С. С., О классификации алгебр со знакопеременной группой автоморфизмов. Докл. AH CCCP (1982) 265, №3, 533-536.

4. Марченков С. С., Классификация алгебр со знакопеременной группой автоморфизмов. Матем. вопросы киберн. (1989) 2, 100-122.

5. Machida H., Miyakawa M., Rosenberg I. G., Relations between clones and full monoids. Proc. 31 Int. Symp. Multiple-Valued Logic. Warsaw, 2001, 279-284.

6. Марченков С. С., О замкнутых классах самодвойственных функций многозначной логики. Проблемы киберн. (1979) 36, 5-22.

7. Марченков С. С., Об однородных алгебрах. Докл. АН СССР (1981) 256, №4, 787-790.

8. Марченков С. С. Однородные алгебры. Проблемы киберн. (1982), 39, 85-106.

9. Нгуен Ван Хоа, О семействах замкнутых классов $k$-значной логики, сохраняемых всеми автоморфизмами. Дискретная математика (1993) 5, №4, 87-108.

10. Нгуен Ван Хоа, Описание замкнутых классов, сохраняемых всеми внутренними автоморфизмами. Докл. АН Беларуси (1994) 38, №3, 16-19.

11. Марченков С. С., Деметрович Я., Ханнак Л., О замкнутых классах самодвойственных функций в $P_{3}$. Методы дискретного анализа в решении комбинаторных задач (1980) 34, 38-73.

12. Demetrovics J., Hannak L., Marchenkov S. S., On closed classes of selfdual functions. Colloquia mathematica societatis Janos Bolyai (1979) 28, 183-189.

13. Demetrovics J., Hannak L., Marchenkov S. S., Some remarks on the structure of $P_{3}$. C. R. Math. Rep. Acad. Sci. Canada (1980) 11, №4, 215-219.

14. Яблонский С. В., Введение в дискретную математику. Наука, Москва, 1986.

15. Боднарчук В. Г., Калужнин Л. А., Котов В. Н., Ромов Б. А., Теория Галуа для алгебр Поста. Кибернетика (1969), №3, 1-10; №5, 1-9.

16. Марченков С. С., О замкнутых классах самодвойственных функций многозначной логики II. Проблемы киберн. (1983) 40, 261-266.

17. Марченков С. С., $A$-замкнутые классы многозначной логики, содержащие константы. Дискретная математика (1998) 10, №3, 10-26.

18. Марченков С. С., А-классификация функций многозначной логики. Докл. $Р A H(1999)$ 366, №4, $455-457$. 